\title{
El software libre: ¿fin de la propiedad individual?
}

\author{
Bernardo Andrés Peralta Pardo; Ilber Darío Saza Garzón²; Víctor Andrés Heredia Heredia ${ }^{3}$
}

Recibido: 26 de febrero de 2015 Aprobado: 25 de mayo de 2015

\begin{abstract}
Resumen
Las tecnologías de la información y las comunicaciones (TIC) han brindado la posibilidad de obtener información de todo tipo, incluyendo software, obras musicales, textos y libros, entre otros. En internet se encuentra software con diversidad de licencias, desde las que permiten libre desarrollo y publicación hasta aquellas con derechos corporativos, que se pueden descargar en sitios web legales y oficiales, e incluso en otros no autorizados que ofrecen el software licenciado con crack para que se pueda utilizar sin ninguna restricción. Por lo anterior, se puede decir que la cultura y la ética del ciudadano digital es la que en últimas define el criterio con el que los usuarios utilizan la información, por lo que surgen varios interrogantes: ¿es posible proteger la información que se genera?, ¿qué pasa con los documentos que se publican en la red?, ¿si alguien reproduce información o software es ilegal? En tal sentido, este artículo se propone ofrecer la información necesaria que le permita al creador de software proteger la propiedad intelectual y los derechos adquiridos a partir de patentes y licencias, que admitan tanto la reescritura como la reproducción, sin desconocer al primer autor o desarrollador.
\end{abstract}

Keywords: Creative Commons, software libre, licencia, freeware, shareware.

\section{Abstract}

Nowadays the technologies of information and communication technology (ICT) have given the possibility of information of all kinds, including software, musical works, texts and books, among others. On the Internet is software with a variety of licenses, from which allow free development $t$ and publication as those graduates with rights corporate, from licensed for free development and publication to graduate with corporate rights (of pay), the latter can be downloaded from official and legal websites, but also of other unauthorized offering software licensed with crack so it can be used without any restriction. For all of the above is that the culture and ethics of the digital citizen is what in latest defines the criteria with which users use the information, so there are several questions, such as: is it possible to protect the information I genre?, what about the documents I publish on the net?, if I play information or software is illegal? In this sense, this article seeks to provide necessary information enabling the creator of software to protect intellectual property and rights acquired from patents and different types of licenses, which allow both rewriting and playback, without disregarding the first author or developer

Keywords: Creative Commons, license, freeware, shareware.

\footnotetext{
1 Bapardo@gmail.com. Ingeniero de sistemas. Especialista y Magíster en Tecnologías de la Información aplicadas a la Educación. Universidad Pedagógica Nacional (UPN).

${ }^{2}$ Dariosaza82@gmail.com. Licenciado en Informática. Especialista y Magíster en Tecnologías de la Información aplicadas a la Educación. Universidad Pedagógica Nacional (UPN).

3 Vaherediah@gmail.com. Licenciado en Física. Especialista y Magíster en Tecnologías de la Información aplicadas a la Educación. Universidad Pedagógica Nacional (UPN).
} 


\section{ELSOFTWARE LIBREY CREATIVE COMMONS}

En este artículo se pretende reflexionar, en primer lugar, sobre cómo se genera el proceso de adquisición de conocimiento y la forma en la que en la historia la humanidad ha demostrado que uno de los medios para obtenerlo es la observación y la imitación. En segundo lugar, el conocimiento generado se puede proteger y llevar a todas las personas. Algunos autores como Albert Bandura, citado por Schunk (1997), lo llaman aprendizaje vicario o por observación. En su teoría, grosso modo, afirma que desde la niñez se imita para aprender (¿sería copiar?).

Ahora bien, en este escrito se incluyen algunos antecedentes en cuanto a cómo la necesidad de copiar e imitar para aprender y conocer ha sido una constante en el ser humano. Por ejemplo, en la Edad Media los escribanos (personas dedicadas a la escritura), hacían copias de textos y pergaminos, pero este acto tenía un costo alto que podía comprometer hasta la propia vida, lo cual recuerda el periodo de la inquisición, en el que se perseguía a quienes innovaban o rompían las barreras del conocimiento. Se atemorizaba a la humanidad para que no estuviera en contra de los dogmas de la Iglesia católica; sin embargo, a pesar de los castigos, siempre hubo personas dispuestas a poner el conocimiento al alcance de todos.

Luego surgió la imprenta, hacia el año de 1450. Creada por Johannes Gutenberg, fue la máquina que revolucionó la forma de copiar, por cuanto permitió optimizar el proceso en cuanto a tiempo y calidad. Así comenzó un crecimiento acelerado de la creación y copia de información tanto de manera legal como ilegal; sin embargo, la reproducción no autorizada de las obras no era tan amplia debido a que los costos que implicaba sostener una imprenta eran altos y muy pocas personas podían asumirlos.

Mucho tiempo después se inventaron los computadores, que constituyeron una nueva forma de crear información y guardarla en cintas magnéticas para llegar a dispositivos de almacenamiento plástico y eléctrico como los CD y las memorias flash.

En la modernidad, con la aparición de los transistores, procesadores y microprocesadores y, en consecuencia, los computadores, surgió una nueva industria tecnológica, informática y digital que produjo un desarrollo notable del sector tanto en la forma física (hardware) como en la lógica (software). En esencia, la existencia de dispositivos le permiten al usuario realizar copia de sus archivos. Al mismo tiempo, esto ha hecho que la copia no autorizada de material, bien sea para uso personal o distribución legal o ilegal, crezca de manera exponencial.
Por otra parte, gracias a las nuevas tecnologías se han producido diversas formas de socialización, y se han concretado conceptos como los de ciberespacio y cibercultura, que son espacios que les permiten a los usuarios compartir vivencias, conocimientos y creaciones. Claro, esto también trae consigo problemas para los creadores, ya que todos sus trabajos se comparten en la web (con o sin consentimiento del autor).

Al desarrollar e innovar con productos informáticos, los autores dedican tiempo, dinero y conocimiento y, en consecuencia, generan trabajo, por lo que buscan un beneficio económico y reconocimiento. En este punto surge la duda de si realmente todos los usuarios potenciales pueden adquirir esos productos de forma legal. La respuesta es que muchos sí lo hacen porque sus ingresos económicos se lo permiten; en otros casos, aunque tengan el poder adquisitivo, por cultura o por ahorrar dinero no lo hacen. Finalmente, hay usuarios que necesitan el producto pero no tienen dinero. En los dos últimos casos se requieren nuevas opciones entre usuarios y autores para evitar sanciones legales por piratería, plagio y derechos de autor.

Además, la necesidad creciente en el usuario de actualizarse e informarse le exige mejorar la tecnología que emplea, por ejemplo el software, que está en constante evolución y actualización, y por ende demanda mejores máquinas, lo que implica dos gastos económicos para el usuario: la actualización del hardware y la compra de licencias. Estos costos en algunos casos son excesivos para los usuarios, lo cual es evidente en los países en vías de desarrollo.

Los usuarios de software buscan soluciones inmediatas para evitar altos costos y en algunos casos prefieren utilizar copias ilegales. Para esto visitan lugares donde las descargan y se benefician económicamente, ya que el único costo que tienen es el del medio de copia. Además, existen otros medios para conseguir software ilegal, como las comunidades con las que se comparte todo tipo de programas de computador, lo que convierte este acto en una cadena.

Por estos problemas que se centran en lo económico y lo cultural, es necesario plantear soluciones que beneficien a ambos actores. De ahí surgió la necesidad en un momento histórico de crear nuevas opciones para el desarrollo y uso de software.

\section{II. ¿QUIÉNES DESARROLLAN SOFTWARE LIBRE?}

El término software libre es un desarrollo tecnológico intangible que cuenta con gran acogida en el contexto académico por generar comunidades de conocimiento 
en el desarrollo de tecnología. Se le prefiere sobre todo por sus bajos costos de distribución y uso. Según Dávila (2009), "El software libre entró en el ámbito de la tecnología con una fuerza tan grande... a pesar de no haber nacido de un movimiento comercial sino de las comunidades académicas". El término software libre hace referencia a la libertad de copiar, reproducir o modificar un programa; no obstante, comúnmente se toma erradamente, por la traducción literal del inglés free (gratis), aunque ésta es sólo una minúscula parte de lo que realmente abarca el término libre.

La primera persona en introducir el término free software fue Richard Stallman, quien en los años setenta, al verse en la necesidad de conocer el código fuente para solucionar un problema con su impresora, no pudo acceder a él y decidió desarrollar un software de uso general, no privativo, y fundó la Free Software Foundation (FSF) para promocionar la distribución de software con su respectivo código fuente.

Es así como el software libre lo desarrollan personas que procuran solucionar una necesidad específica ya sea de forma individual o cooperativa, como es el caso de sistemas operativos que se distribuyen de forma libre, entre los cuales el más popular es Linux, creado inicialmente por Linus Torvalds. Éste es un sistema operativo de distribución gratuita y código abierto, lo que significa que cualquier persona con conocimientos de programación puede colaborar en su desarrollo.

El sistema operativo Linux se ha hecho muy popular entre los usuarios, tanto así que es la principal competencia de la multinacional Microsoft y su sistema operativo Windows ${ }^{\circledR}$, lo que demuestra que el mercado está abierto a todo tipo de productos, sean de distribución libre o paga. En ese sentido hay muchas compañías que realizan software de libre distribución, y han llevado sus mercados a otros contextos como el de servicios.

Por otro lado, el software libre no sólo lo desarrollan personas naturales. También se crea en el ámbito corporativo. Es el caso de Skype, desarrollado por el sueco Niklas Zennström y el danés Janus Friiswhose, que aun cuando es de libre distribución y descarga gratuita, posee algunas funciones pagas, como las llamadas a teléfonos móviles y fijos alrededor del mundo. Sin embargo, ésta es una pequeña parte de todo lo que permite la herramienta, puesto que admite, entre otras cosas, hacer llamadas de voz y video sólo con tener acceso a internet.

En Colombia se puede citar como ejemplo de software libre el sistema de gestión de datos Orfeo, desarrollado por la Superintendencia de Servicios Públicos, que actualmente está siendo implementado por más entidades estatales.
Uno de los principales argumentos de los detractores de este tipo de software es la pérdida de la propiedad intelectual del producto en manos de terceros, así como los derechos que se puedan derivar del mismo. No obstante, existen múltiples tipos de licencias como GNU, GPL, LGPL, entre otras, lo importante es que, si bien se distribuyen a gran escala y sin ningún tipo de control, es posible mantener la propiedad intelectual y garantizar su libre distribución, sin que terceros se lucren del producto o lo registren mediante licencia comercial.

Para garantizar y promover este tipo de producciones existen numerosas organizaciones en el mundo que incentivan y crean proyectos de divulgación de desarrollos libres, al igual que comunidades colaborativas en proyectos comunes orientadas a popularizar esta cultura. En Colombia algunas fundaciones dedicadas al desarrollo o protección del software libre, entre las cuales sobresale la Fundación Software Libre Colombia (FSLCOL), dedicada a la promoción e implementación de tecnologías basadas en estándares abiertos.

Ahora bien, múltiples licencias protegen el desarrollo de software libre, y cada una permite cierto nivel de reproducción del material, como se expondrá a continuación.

\section{LICENCIAS TIPO GNU/GPL}

Según Stallman (2013), el origen de las licencias GNU / GPL es el siguiente:

GNU es un acrónimo recursivo que significa "GNU No es Unix" (GNU isNot Unix). G: GNU is N: Not U: Unix. Debido a que se pronuncia muy similar a "ñu", su creador, Richard Stallman, decidió simbolizarlo con este animal africano.

Se le dio el nombre de GNU al primer proyecto que trató de desarrollar un sistema operativo libre. Por eso sus siglas se asocian a la licencia de tipo GPL (General Public License).

En 1991, Linus Torvalds liberó el núcleo Linux con los términos de la GPL, completando un sistema GNU completo y operativo: el GNU/Linux. Ahora bien las licencias GNU/GPL están definidas por Stallman (2013) de la siguiente forma:

- La licencia pública general de GNU, más conocida por su nombre en inglés GNU General Public License o simplemente sus siglas del inglés GNU GPL, fue creada por la Free Software Foundation en 1989 (la primera versión, escrita por Richard Stallman), está orientada principalmente a proteger la libre distribución, modificación y uso de software. Su propósito es declarar que el software cubierto por esta licencia 
es libre y protegerlo de intentos de apropiación que restrinjan esas libertades a los usuarios.

- Existen varias licencias "hermanas" de la GPL, como la de documentación libre de GNU (GFDL) y la Open Audio License para trabajos musicales, entre otras.

- Copyleft: se practica al ejercer el derecho de autor, que consiste en permitir la libre distribución de copias y versiones modificadas de una obra u otro trabajo, exigiendo que los mismos derechos se preserven en las versiones modificadas.

\section{RELACIÓNENTRE CREATIVE COMMONS (CC)} Y SOFTWARE LIBRE

Las licencias Creative Commons (CC) surgen de Creative Commons Organization, entidad internacional sin ánimo de lucro creada por Lawrence Lessig, cuyo objetivo es ofrecer un esquema legal de licencias y aplicaciones informáticas que permitan la circulación y el uso de contenidos de manera pública, con el fin de que los autores definan los términos en que sus obras se pueden usar, qué derechos desean entregar y en qué condiciones (Creative Commons Colombia, 2011). Al contrario del sistema tradicional correspondiente al derecho de autor o Copyright "todos los derechos reservados", estas licencias protegen "algunos derechos reservados".

Alrededor del proyecto CC se siguen sumando numerosos participantes entre los que se encuentran profesores, creadores, editores y estudiosos que tomando como referencia las experiencias de producción libre y cooperativa del software libre, pretenden trasladar estas mismas ideas a otros sectores de la producción intelectual.

Según Creative Commons Colombia (2011), en el año 2001:

“...la General Public License (GPL), concebida por la Free Software Foundation (FSF), llevaba unos cuantos años de funcionamiento demostrando precisamente como un aprovechamiento de las restrictivas leyes sobre propiedad intelectual se pueden emplear para promocionar la copia, uso, modificación y distribución de contenidos software en pro del enriquecimiento cultural de toda la comunidad".

CC nace a partir del seguimiento de este tipo de iniciativas pioneras, planteando el desafío de la extensión de este tipo de filosofías de producción colaborativa, descentralizada y abierta a otras áreas de producción como el de las obras artísticas, culturales, científicas y educativas.

Así, pues, entre los objetivos primordiales de CC no se encuentran el recoger licencias que amparen los programas software. De hecho, para el caso del software CC recomienda explícitamente el uso de las licencias ofrecidas por la FSF y la Open Source Initiative. Debemos considerar por tanto las licencias CC como un complemento, y no como un sustituto, de las licencias del mundo del software libre, puesto que de hecho están orientadas a otros entornos de creación".

No obstante, algunos de los creadores de software pueden plantear la utilización de licencias CC para distribuir la documentación que acompaña a sus programas. También se puede pensar en creadores de contenidos (imágenes, sonidos, video o texto), interesados en licenciarlos en alguna de las modalidades ofrecidas por CC para su inclusión en paquetes de software libre.

Se puede indicar que las licencias tipo CC no aplican para el software libre ya que fueron creadas con otros fines. Si se refiere al uso del software sólo se puede encontrar dos tipos básicos de licencia: el copyright y las de tipo GPU/GPL; las primeras se utilizan para el software de uso privativo o licenciado; y las segundas, como se explica en un apartado anterior, para software libre. Así que las licencias CC se destinan principalmente a obras literarias, artículos, fotografías, obras de arte, entre otras y no para software.

\section{REFERENCIAS}

1. Creative Commons. Creative Commons Colombia. (n.d.). Retrieved from. Recuperado de: http:// co.creativecommons.org/?page_id=12.

2. Dávila, M. (2009) GNU/Linux y el software libre y sus múltiples aplicaciones. Bogotá. Editorial AlfaOmega.

3. Dávila, M., (2010), Software libre, una visión. Computerworld Colombia, Uniminuto. Creative Commons Colombia. Recuperado de http:// co.creativecommons.org/quienes-somos/.

4. De Ferias, E. (2005). Algunos casos de piratería en internet. Pensar en Libro, Cerlalc, Unesco, edición N. ${ }^{\circ} 2$. 
5. Etxeberria, A. (2008). Software libre. Escuela Virtual, UN DP.

6. Fortino, M. H. (n.d.). Libros y artículos sobre software libre y open source. Recuperado de http: / /fortinux. com/libros-y-articulos-varios-sobre-software-libre/ libros-y-articulos-sobre-software-libre-y-open-source/.

7. La flecha. (n.d) Recuperado de http://www. laflecha.net/canales/softlibre/articulos/.

8. Skype, historia de una empresa codiciada. (24 de mayo de 2011). Recuperado de http: / /www.elespectador. com/tecnologia/skype-historia-de-una-empresacodiciada-articulo-401618 el 9 de enero 2015.

9. Schunk, Dale H. (1997). Teorías del aprendizaje. México: Pearson Educación.
10. Stallman, R., (2013), The GNU Project. Recuperado de http://www.gnu.org/gnu/thegnuproject. en.html.

11. Stallman, R. (2004). Software libre para una sociedad libre. Traficantes de sueños. The Free Software Foundation. (n.d.). The Free Software Foundation (FSF) is a nonprofit with a worldwide mission to promote computer user freedom and to defend the rights of all free software users. Recuperado de: http:// www.fsf.org.

12. Wikilearning. (n.d). Las licencias Creative Commons - Creative Commons. Recuperado de http://www.wikilearning.com/tutorial/ las_licencias_creative_commons-creative_ commons/6314-2. 